\title{
Observational signatures of past mass-exchange episodes in massive binaries: The case of HD $149404^{\star}, \star \star$
}

\author{
F. Raucq ${ }^{1}$, G. Rauw ${ }^{1}$, E. Gosset ${ }^{1, \star \star \star}$, Y. Nazée ${ }^{1, \star \star \star \star}$, L. Mahy ${ }^{1, \dagger}$, A. Hervé ${ }^{2}$, and F. Martins ${ }^{3}$ \\ ${ }^{1}$ Department of Astrophysics, Geophysics and Oceanography, Liège University, Quartier Agora, Allée du 6 Août 19c, Bat. B5c, \\ 4000 Liège, Belgium \\ e-mail: fraucq@doct.ulg.ac.be \\ 2 Astronomical Institute of the Czech Academy of Sciences, Fričova 298, 25165 Ondřejov, Czech Republic \\ 3 LUPM, Montpellier University 2, CNRS, place Eugène Bataillon, 34095 Montpellier, France \\ Received 12 October 2015 / Accepted 14 January 2016
}

\section{ABSTRACT}

\begin{abstract}
Context. Mass and momentum exchanges in close massive binaries play an important role in their evolution, and produce several observational signatures such as asynchronous rotation and altered chemical compositions, that remain after the stars detach again. Aims. We investigated these effects for the detached massive O-star binary HD 149404 (O7.5 If + ON9.7 I, $P=9.81$ days), which is thought to have experienced a past episode of case A Roche-lobe overflow (RLOF).

Methods. Using phase-resolved spectroscopy, we performed the disentangling of the optical spectra of the two stars. The reconstructed primary and secondary spectra were then analysed with the CMFGEN model atmosphere code to determine stellar parameters, such as the effective temperatures and surface gravities, and to constrain the chemical composition of the components. We complemented the optical study with the study of IUE spectra, which we compare to the synthetic binary spectra. The properties of the stars were compared to evolutionary models.

Results. We confirmed a strong overabundance in nitrogen $\left([\mathrm{N} / \mathrm{C}] \sim 150[\mathrm{~N} / \mathrm{C}]_{\odot}\right)$ for the secondary and a slight nitrogen overabundance $\left([\mathrm{N} / \mathrm{C}] \sim 5[\mathrm{~N} / \mathrm{C}]_{\odot}\right)$ for the primary star. Comparing the two stars, we found evidence for asynchronous rotation, with a rotational period ratio of $0.50 \pm 0.11$.

Conclusions. The hypothesis of a past case A RLOF interaction in HD 149404 is most plausible to explain its chemical abundances and rotational asynchronicity. Some of the observed properties, such as the abundance pattern, are clearly a challenge for current case A binary evolution models, however.
\end{abstract}

Key words. stars: early-type - stars: fundamental parameters - binaries: spectroscopic - stars: individual: HD 149404 - stars: massive

\section{Introduction}

As shown in recent studies (e.g. Sana et al. 2012), a large portion of massive stars belongs to binary or higher multiplicity systems. Whilst this multiplicity permits observationally determining the minimum masses of the stars through their orbital motion, it also leads to complications. For instance, the binarity of massive stars can result in interactions between their stellar winds that produce observational signatures throughout the electromagnetic spectrum (e.g. Rauw 2013). Moreover, during the lifetime of a massive binary, the binarity influences the evolution of the stars in various ways (e.g. Langer 2012). These evolutionary effects range from tidally induced rotational mixing (e.g. de Mink et al. 2009) over exchange of matter and angular momentum through a Roche lobe overflow (RLOF) interaction (e.g. Podsiadlowski et al. 1992; de Loore \& Vanbeveren 1994; Wellstein et al. 2001; Hurley et al. 2002) to the merging of both stars (e.g. Podsiadlowski et al. 1992; Wellstein et al. 2001). In RLOF interactions, three different situations are

\footnotetext{
* Based on observations collected at the European Southern Observatory (La Silla, Chile) and with the International Ultraviolet Explorer.

$\star \star$ The reduced spectra are only available at the CDS via anonymous ftp to cdsarc.u-strasbg. fr $(130.79 .128 .5)$ or via

http://cdsarc.u-strasbg.fr/viz-bin/qcat?J/A+A/588/A10

$\star \star \star$ Senior Research Associate F.R.S-FNRS.

$\star \star \star \star$ Research Associate F.R.S-FNRS

$\dagger$ Postdoctoral Researcher F.R.S-FNRS.
}

distinguished: case A, for which the RLOF episode occurs when the mass-donor is on the core-hydrogen burning main-sequence; case $\mathrm{B}$, when the star is in the hydrogen-shell burning phase; and case $\mathrm{C}$, for which the star is in the helium-shell burning phase (Kippenhahn \& Weigert 1967; Vanbeveren et al. 1998). These binary interactions significantly affect the physical properties of the components and their subsequent evolution. Despite considerable progresses in theoretical models, there remain a number of open questions such as the actual efficiency of accretion (e.g. Wellstein et al. 2001; de Mink et al. 2007; Dray \& Tout 2007). To better understand this phenomenon, we need in-depth studies of systems undergoing or having undergone mass exchange.

In this context, HD 149404 is an interesting target. It is a detached, non-eclipsing O-star binary, member of the Ara OB1 association, located at a distance of $1.3 \pm 0.1 \mathrm{kpc}$ (Herbst \& Havlen 1977). The orbital parameters were revised by Rauw et al. (2001). These authors found an orbital period of 9.81 days and a circular orbit. They also estimated an orbital inclination of $21^{\circ}$ by comparing "typical" masses of supergiants with similar spectral types to those of both components. The spectrum of HD 149404 displays variable emission lines (He II $\lambda$ 4686, $\mathrm{H} \alpha$ ) that are likely indicative of a wind-wind interaction (Rauw et al. 2001; Thaller et al. 2001; Nazé et al. 2002). One of its components was assigned an ON spectral type, which suggests a significant nitrogen enrichment of its atmosphere.

In this study, we determine the fundamental parameters of the stars of this system through several analysis techniques. The data used in our study are presented in Sect. 2. In Sect. 3 
we present the preparatory treatment of our data, which included disentangling the spectra of the binary system to reconstruct individual spectra of the binary components, which are needed for the subsequent spectral analysis. The spectral analyses were made with the non-LTE model atmosphere code CMFGEN and are presented in Sect. 4. In Sect. 5 we discuss the evolutionary state of HD 149404 and compare it with similar systems.

\section{Observational data}

The optical spectra analysed here are the échelle spectra used by Rauw et al. (2001) in their study of the orbital solution and wind interactions of HD 149404. These data were collected in May 1999 and February 2000 with the FEROS and Coralie spectrographs mounted on the ESO $1.5 \mathrm{~m}$ telescope and $1.2 \mathrm{~m}$ Euler Swiss telescope at La Silla, respectively. The FEROS and Coralie spectrographs have resolving powers of 48000 and 50000 and cover the wavelength ranges 3650-9200 and 3875-6800 $\AA$, respectively. Further details on the instrumentation and the data reduction are given by Rauw et al. (2001). Because of some problems with the extraction of the FEROS data of May 2000, the normalization of the latter was more uncertain, and we therefore excluded these data from the disentengling procedure, and thus from the further analysis.

To complement our optical data, we also retrieved 25 shortwavelength, high-resolution (SWP) IUE spectra of HD 149404 from the IUE archives. These spectra were previously described by Stickland \& Koch (1996).

Table 1 yields the journal of observations along with the orbital phases computed according to the ephemerides of Rauw et al. (2001, their He I $\lambda 4471$ orbital solution). The optical spectra provide a good sampling of the orbital cycle, which is important for the disentangling in Sect. 3 .

\section{Preparatory analysis}

\subsection{Spectral disentangling}

The previous determination of the orbital solution of the system performed by Rauw et al. (2001) allowed us to recover the individual spectra of both components by separating the normalized spectra of the binary system. For this purpose, we used our disentangling routine (Rauw 2007), which is based on the method of González \& Levato (2006) and was previously used by Linder et al. (2008) and improved by Mahy et al. (2012). The basic idea behind this technique is that the most natural way to handle spectra for radial velocity measurements is to express them as a function of $x=\ln \lambda$ instead of as a function of $\lambda$. In this way, the Doppler shift for a line of rest wavelength $\lambda_{0}$ can be expressed as

$$
\begin{aligned}
\ln \lambda & =\ln \left[\lambda_{0}\left(1+\frac{v}{c}\right)\right] \\
& =\ln \lambda_{0}+\ln \left(1+\frac{v}{c}\right) \\
& \simeq \ln \lambda_{0}+\frac{v}{c} \\
\Rightarrow x & \simeq x_{0}+\frac{v}{c}
\end{aligned}
$$

Let $A(x)$ and $B(x)$ be the spectra of the primary and secondary in the heliocentric frame of reference. In the absence of line profile variability and eclipses, the spectrum observed at any phase $\phi$ can be written as

$$
S(x)=A\left(x-\frac{v_{\mathrm{A}}(\phi)}{c}\right)+B\left(x-\frac{v_{\mathrm{B}}(\phi)}{c}\right),
$$

\begin{tabular}{|c|c|c|c|c|}
\hline \multicolumn{5}{|c|}{ Optical spectroscopy } \\
\hline \multicolumn{2}{|c|}{ HJD-2 450000} & Inst. & $\phi$ & \\
\hline \multicolumn{2}{|l|}{1299.796} & FEROS & 0.24 & \\
\hline \multicolumn{2}{|l|}{1300.788} & FEROS & 0.34 & \\
\hline \multicolumn{2}{|l|}{1301.792} & FEROS & 0.45 & \\
\hline \multicolumn{2}{|l|}{1302.783} & FEROS & 0.55 & \\
\hline \multicolumn{2}{|l|}{1304.792} & FEROS & 0.75 & \\
\hline \multicolumn{2}{|l|}{1578.866} & Coralie & 0.68 & \\
\hline \multicolumn{2}{|l|}{1579.879} & Coralie & 0.78 & \\
\hline \multicolumn{2}{|c|}{1580.872} & Coralie & 0.88 & \\
\hline \multicolumn{2}{|c|}{1581.864} & Coralie & 0.98 & \\
\hline \multicolumn{2}{|l|}{1582.869} & Coralie & 0.09 & \\
\hline \multicolumn{2}{|c|}{1583.877} & Coralie & 0.19 & \\
\hline \multicolumn{2}{|l|}{1584.857} & Coralie & 0.29 & \\
\hline \multicolumn{2}{|c|}{1585.845} & Coralie & 0.39 & \\
\hline \multicolumn{2}{|l|}{1586.870} & Coralie & 0.49 & \\
\hline \multicolumn{2}{|c|}{1587.861} & Coralie & 0.59 & \\
\hline \multicolumn{2}{|l|}{1588.886} & Coralie & 0.70 & \\
\hline \multicolumn{2}{|c|}{1590.886} & Coralie & 0.90 & \\
\hline \multicolumn{5}{|c|}{ IUE SWP spectroscopy } \\
\hline 2440000 & $\phi$ & \multicolumn{2}{|c|}{ JD-2 440000} & $\phi$ \\
\hline 8.044 & 0.76 & \multicolumn{2}{|c|}{4450.176} & 0.35 \\
\hline 4.588 & 0.42 & \multicolumn{2}{|c|}{4460.090} & 0.36 \\
\hline 6.869 & 0.75 & \multicolumn{2}{|c|}{9564.333} & 0.42 \\
\hline 6.921 & 0.76 & \multicolumn{2}{|c|}{9565.329} & 0.52 \\
\hline 6.966 & 0.76 & \multicolumn{2}{|c|}{9817.595} & 0.23 \\
\hline 3.028 & 0.40 & \multicolumn{2}{|c|}{9818.630} & 0.33 \\
\hline 8.965 & 0.00 & \multicolumn{2}{|c|}{9840.402} & 0.55 \\
\hline 26.010 & 0.95 & \multicolumn{2}{|c|}{9910.425} & 0.68 \\
\hline 1.156 & 0.60 & \multicolumn{2}{|c|}{9911.396} & 0.78 \\
\hline 1.203 & 0.61 & 9912. & & 0.88 \\
\hline 0.089 & 0.72 & 9913. & & 0.99 \\
\hline 29.216 & 0.65 & 9915. & & 0.19 \\
\hline 2.327 & 0.50 & & & \\
\hline
\end{tabular}

A10, page 2 of 11
Table 1. Journal of the optical (top) and UV (bottom) spectra.

where $v_{\mathrm{A}}(\phi)$ and $v_{\mathrm{B}}(\phi)$ are the radial velocities of stars $\mathrm{A}$ and $\mathrm{B}$ at orbital phase $\phi$.

For a total of $n$ observed spectra, the spectrum of the primary can be computed as the average of the observed spectra from which the secondary spectrum shifted by the appropriate radial velocity (RV) has been subtracted (González \& Levato 2006) :

$A(x)=\frac{1}{n} \sum_{i=1}^{n}\left[S_{i}\left(x+\frac{v_{\mathrm{A}}\left(\phi_{i}\right)}{c}\right)-B\left(x-\frac{v_{\mathrm{B}}\left(\phi_{i}\right)}{c}+\frac{v_{\mathrm{A}}\left(\phi_{i}\right)}{c}\right)\right]$.

And similarly for the secondary:

$B(x)=\frac{1}{n} \sum_{i=1}^{n}\left[S_{i}\left(x+\frac{v_{\mathrm{B}}\left(\phi_{i}\right)}{c}\right)-A\left(x-\frac{v_{\mathrm{A}}\left(\phi_{i}\right)}{c}+\frac{v_{\mathrm{B}}\left(\phi_{i}\right)}{c}\right)\right]$.

Starting from an initial approximation for $B(x)$ (a flat spectrum), $v_{\mathrm{A}}\left(\phi_{i}\right)$ and $v_{\mathrm{B}}\left(\phi_{i}\right)$, we then iteratively determined the primary and secondary spectra and their radial velocities (see González \& Levato 2006). For the separations we performed here, we fixed the radial velocities of the binary components to those corresponding to the orbital solution of Rauw et al. (2001).

As for any disentangling method, this technique also has its limitations (González \& Levato 2006). A reasonable observational sampling of the orbital cycle is needed because the quality of the results depends on the radial velocity (RV) ranges covered by these observations. Another important limitation is that broad spectral features are not recovered with the same accuracy as narrow ones. This is the case for the wings of the Balmer 
Table 2. Brightness ratio determination from the dilution of prominent lines.

\begin{tabular}{l|ccccccc}
\hline \hline Line & \multicolumn{6}{|c}{ Equivalent width $(\AA)$} & \multicolumn{3}{c}{$l_{1} / l_{2}$} \\
\hline & \multicolumn{3}{|c}{ Observations } & \multicolumn{2}{c}{ Synthetic spectra } & Conti $(1973,1974)$ \\
\hline & Primary & Secondary & O7.5 & O9.7 & O7.5 & O9.5 \\
\hline He I $\lambda 4026$ & 0.19 & 0.34 & 0.72 & 0.74 & 0.65 & 0.80 & 0.57 \\
Si IV $\lambda 4089$ & 0.12 & 0.34 & 0.32 & 0.56 & 0.25 & 0.53 & 0.61 \\
Si IV $\lambda 4116$ & 0.06 & 0.24 & 0.11 & 0.29 & 0.12 & 0.37 & 0.66 \\
He II $\lambda 4200$ & 0.11 & 0.08 & 0.47 & 0.21 & 0.42 & 0.23 & 0.61 \\
H $\gamma$ & 0.45 & 0.50 & 1.77 & 1.74 & 1.88 & 2.09 & 0.88 \\
He I $\lambda 4471$ & 0.17 & 0.37 & 0.55 & 0.69 & 0.71 & 0.97 & 0.58 \\
He II $\lambda 4542$ & 0.17 & 0.07 & 0.53 & 0.21 & 0.64 & 0.25 & 0.96 \\
\hline
\end{tabular}

Notes. The measured EWs are compared with values for the same lines in synthetic spectra of the same spectral type and in the compilation of measurements from the literature. The last column yields the brightness ratio for each line considered, using the synthetic spectra EWs.

lines, but it also holds for the UV features that arise in the stellar winds, such as P-Cygni profiles. For instance, for the IUE spectra of HD 149 404, the Si IV $\lambda 1393-1403$ and N IV $\lambda 1718$ PCygni lines are very broad and barely vary during the orbital cycle. It was therefore impossible to properly separate the primary and secondary contributions for these lines. In practice, features that are wider than a few times the RV amplitude are barely recovered. Moreover, small residual errors in the normalization of the input spectra can lead to oscillations of the continuum in the resulting separated spectra on wavelength scales of several dozen $\AA$. Finally, spectral disentangling works on continuumnormalized spectra and does not yield the brightness ratio of the stars, which must be determined by other techniques (see below).

Finally, in the specific case of HD 149404, several emission lines ( $\mathrm{H} \alpha$ and $\mathrm{He}$ II $\lambda$ 4686, for example) do not seem to arise in the atmosphere of either component, but are formed at least partly in the wind-wind interaction zone (Rauw et al. 2001; Thaller et al. 2001; Nazé et al. 2002). As a result, the disentangling code is unable to meaningfully reconstruct these lines in the spectra of the components. We need to keep this in mind when analysing the reconstructed spectra.

\subsection{Spectral types}

Based on the reconstructed individual line spectra of the primary and secondary components, we determined the spectral types of the stars by measuring the equivalent width ratio of the spectral lines He I $\lambda 4471$ and He II $\lambda 4542$ on the one hand, and Si IV $\lambda 4088$ and He I $\lambda 4143$, on the other hand. We applied Conti's quantitative classification criteria for O-type stars (Conti \& Alschuler 1971; Conti \& Frost 1977; see also van der Hucht 1996) for both spectral types and luminosity classes to determine that the primary star is an O7.5 If star, while the secondary is an ON9.7 $\mathrm{I}^{1}$. Since our classification is based on the separated spectra, it is less sensitive to a possible phase-dependence of the line strengths and should thus be more robust than a classification based on spectra that are only collected near quadrature phase, as done by Rauw et al. (2001). However, in this particular case, we find an excellent agreement with the classification $(\mathrm{O} 7.5 \mathrm{I}(\mathrm{f})+\mathrm{ON} 9.7 \mathrm{I})$ proposed by these authors.

\footnotetext{
1 In the literature on interacting binaries, the terminologies "primary" and "secondary" usually designate the initially more massive star and less massive star, respectively. However, in accordance with previous studies of the HD 149404 system, in the present paper the terms "primary" and "secondary" star refer to the currently more massive and less massive component, respectively.
}

\subsection{Brightness ratio}

Spectral disentangling yields the strength of the lines in the primary and secondary stars relative to the combined continuum, but does not allow establishing the relative strengths of the continua. To further analyse the separated spectra, we therefore need to first establish the brightness ratio of the stars.

To estimate the optical brightness ratio of the components of HD 149404, we measured the equivalent widths of a number of spectral lines on the separated spectra of the primary and secondary, but referring to the combined continuum of the two stars. The results are listed in Table 2 along with the mean equivalent widths of the same lines in synthetic spectra of stars of the same spectral type, computed with the non-LTE model atmosphere code CMFGEN (Hillier \& Miller 1998), which we describe in Sect.4.2. For comparison, we also list the mean equivalent widths of the same lines in spectra of stars of the same spectral type as evaluated from the measurements of Conti (1973, 1974) and Conti \& Alschuler (1971). from

The brightness ratio of the two stars can then be evaluated

$\frac{l_{1}}{l_{2}}=\left(\frac{E W_{1}}{E W_{2}}\right)_{\text {obs }}\left(\frac{E W_{O 9.7}}{E W_{O 7.5}}\right)_{\text {mean }}$.

By combining our measurements with those from synthetic spectra, we derive an optical brightness ratio of $0.70 \pm 0.12$ in this way, which is slightly lower than the value found by Rauw et al. (2001), who inferred $0.90 \pm 0.16$. As a consistency check, we also determined the brightness ratio by comparison with Conti's measurements and obtained $0.79 \pm 0.13$. The difference between the results obtained based on synthetic spectra versus Conti's measurements arises at least partially because Conti (1973, 1974) did not take into account the spectral type 09.7, but instead considered an 09.5 spectral type, consisting of both classes of stars currently known as 09.5 and 09.7.

Reed (2003) reported a mean $V$ magnitude of $5.48 \pm 0.02$ and $B-V$ colour of $0.39 \pm 0.02$ for the system. Since the intrinsic $(B-V)_{0}$ of an 07.5 or O9.5 star is -0.26 (Martins \& Plez 2006), the extinction $A_{V}$ can be calculated as being $2.02 \pm 0.06$, assuming $R_{V}=3.1$. Assuming the $1.3 \pm 0.1 \mathrm{kpc}$ distance (Wolk et al. 2008), we infer an absolute $M_{V}=-7.11 \pm 0.07$ for the binary. A brightness ratio of $l_{1} / l_{2}=0.70 \pm 0.12$ then yields individual absolute magnitudes of $M_{V}^{P}=-6.15 \pm 0.13$ and $M_{V}^{S}=-6.53 \pm 0.10$.

The separated continuum-normalized primary and secondary optical spectra are shown and analysed in Fig. 1, in Sect. 4.4. 
Table 3. Projected rotational velocities $\left(v \sin i\right.$ in $\left.\mathrm{km} \mathrm{s}^{-1}\right)$ of the components of HD 149404.

\begin{tabular}{l|cc}
\hline \hline Line & Primary & Secondary \\
\hline He I $\lambda 4026$ & 97 & 75 \\
He I $\lambda$ 4922 & 94 & 64 \\
He I $\lambda 5016$ & 105 & 58 \\
O III $\lambda$ 5592 & 87 & 55 \\
C IV $\lambda$ 5812 & 82 & - \\
\hline Mean value & $93 \pm 8$ & $63 \pm 8$ \\
\hline
\end{tabular}

\section{Spectral analysis}

\subsection{Rotational velocities and macroturbulence}

After applying a Fourier transform method (Simón-Díaz \& Herrero 2007; Gray 2008) to the profiles of the He I $\lambda \lambda 4026$, 4922, 5016, O III $\lambda$ 5592, and C IV $\lambda 5812$ lines of the separated spectra, we determined the projected rotational velocities $v \sin i$ of the stars of the system. We selected these lines because they are very well isolated in the spectra and are therefore expected to be free of blends. The results are presented in Table 3 . The mean $v \sin i$ of the primary star is $(93 \pm 8) \mathrm{km} \mathrm{s}^{-1}$, while that of the secondary is $(63 \pm 8) \mathrm{km} \mathrm{s}^{-1}$.

Macroturbulence is defined as a non-thermal motion in the stellar atmosphere in which the size of the turbulent cell is greater than the mean free-path of the photons. The main effect of the macroturbulence is an additional broadening of the spectral lines. This effect has received much attention over recent years (e.g. Simón-Díaz \& Herrero 2014). A first approximation of the macroturbulence velocities is obtained by applying the radial-tangential anisotropic macroturbulent broadening formulation of Gray (2008) on the spectra after including rotational velocity broadening. We used the auxiliary program MACTURB of the stellar spectral synthesis program SPECTRUM v2.76 developed by Gray (2010). We applied this technique to the lines He I $\lambda \lambda$ 4026, 4471, and 5016, and He II $\lambda$ 4542. In this way, we obtained macroturbulence velocities of 70 and $80 \mathrm{~km} \mathrm{~s}^{-1}$ for the primary and secondary stars, respectively. Although this is quite high, these numbers are consistent with measurements of Simón-Díaz \& Herrero (2014) that were made on stars of similar spectral types.

Both rotational and macroturbulence velocities were applied on the synthetic spectra (see Sect. 4.2) before comparing the latter with the separated spectra.

\subsection{CMFGEN code}

To determine the fundamental properties of the components of HD 149404, we used the non-LTE model atmosphere code CMFGEN (Hillier \& Miller 1998). This code solves the equations of radiative transfer and statistical equilibrium in the co-moving frame for plane-parallel or spherical geometries for Wolf-Rayet stars, O stars, luminous blue variables, and supernovae. CMFGEN furthermore accounts for line blanketing and its effect on the energy distribution. The hydrodynamical structure of the stellar atmosphere is specified as an input to CMFGEN. For the stellar wind, a $\beta$ law is used to describe the velocity law. In the resolution of the equations of statistical equilibrium, a super-level approach is adopted. The following chemical elements and their ions are included in the calculations: $\mathrm{H}$, $\mathrm{He}, \mathrm{C}, \mathrm{N}, \mathrm{O}, \mathrm{Ne}, \mathrm{Mg}, \mathrm{Al}, \mathrm{Si}, \mathrm{S}, \mathrm{Ca}, \mathrm{Fe}$, and $\mathrm{Ni}$. The solution of the equations of statistical equilibrium is used to compute a new photospheric structure, which is then connected to the same $\beta$ wind velocity law. The radiative transfer equations are solved based on the structure of the atmosphere, with a microturbulent velocity varying linearly with velocity from $10 \mathrm{~km} \mathrm{~s}^{-1}$ in the photosphere to $0.1 \times v_{\infty}$ at the outer boundary, and generated synthetic spectra are compared to the separated spectra.

As a first approximation, we assumed that gravity, stellar mass, radius, and luminosity are either assumed equal to typical values of stars of the same spectral type (Martins et al. 2005) or adopted from the study of Rauw et al. (2001). The mass-loss rates and $\beta$ parameters were taken from Muijres et al. (2012) for the spectral types of both stars, whilst the wind terminal velocity was set equal to $2450 \mathrm{~km} \mathrm{~s}^{-1}$ (Howarth et al. 1997) for the two components.

The relevant parameters were then adjusted by an iterative process because each adjustment of a given parameter leads to some modifications in the value of others. This process and the results we obtained are presented in Sects. 4.3 and 4.4.

\subsection{Method}

The first step was to adjust the temperature in the stellar models. The temperatures were mostly determined through the relative strengths of the He I $\lambda 4471$ and He II $\lambda 4542$ lines (Martins 2011). The final values are 34000 and $28000 \mathrm{~K}$ for the primary and secondary stars. Both results agree reasonably well with the temperatures expected for supergiants of these spectral types (Martins et al. 2005).

Subsequently, the natural steps to follow would be adjusting the surface gravities, the mass-loss rate, and the clumping factor, but the binarity of the studied system causes some problems. Indeed, since the wings of broad lines are not properly restored by the disentangling, it is impossible to use the Balmer lines to constrain the surface gravity. And since the $\mathrm{H} \alpha$ and $\mathrm{H} \beta$ lines are in addition strongly affected by extra emission from the windwind interaction (Rauw et al. 2001; Thaller et al. 2001; Nazé et al. 2002), they cannot be used as diagnostics of the massloss rate and clumping factor. The mass-loss rates were therefore fixed to the values tabulated by Muijres et al. (2012) for stars of the same spectral type (see their Table 1, method A). The clumping formalism used in the CMFGEN model is

$f(r)=f_{1}+\left(1-f_{1}\right) \mathrm{e}^{\left(-\frac{V(r)}{f_{2}}\right)}$

with a filling factor $f_{1}$ of 0.1 , a clumping velocity factor $f_{2}$ of $100 \mathrm{~km} \mathrm{~s}^{-1}$, and $V(r)$ the velocity of the wind.

We used an iterative process to constrain the luminosities and surface gravities. The first estimate of $\log (g)$ was taken from Martins et al. (2005) following the spectral types of the stars. Combined with our determination of the effective temperatures, we then inferred the bolometric corrections (Lanz \& Hubeny 2003). This in turn yielded the individual bolometric luminosities, using the absolute $V$ magnitudes of the components derived in Sect. 3.3.

These bolometric luminosities and the effective temperatures were used to compute the ratio of the stellar radii $\frac{R_{\mathrm{P}}}{R_{\mathrm{S}}}$. Together with the assumed gravities, this ratio yields the spectroscopic mass ratio $\frac{M_{\mathrm{P}}}{M_{\mathrm{S}}}$, which was then compared to the dynamical mass ratio inferred from the orbital solution (Rauw et al. 2001). The difference in these mass ratios was used to produce a new estimate of the surface gravities, and this iterative process was repeated until the spectroscopic and dynamical mass ratios agreed with each other and the CMFGEN synthetic spectra produced for the new gravity values matched the observations as well as possible. 
F. Raucq et al.: Signatures of past mass exchange in HD 149404
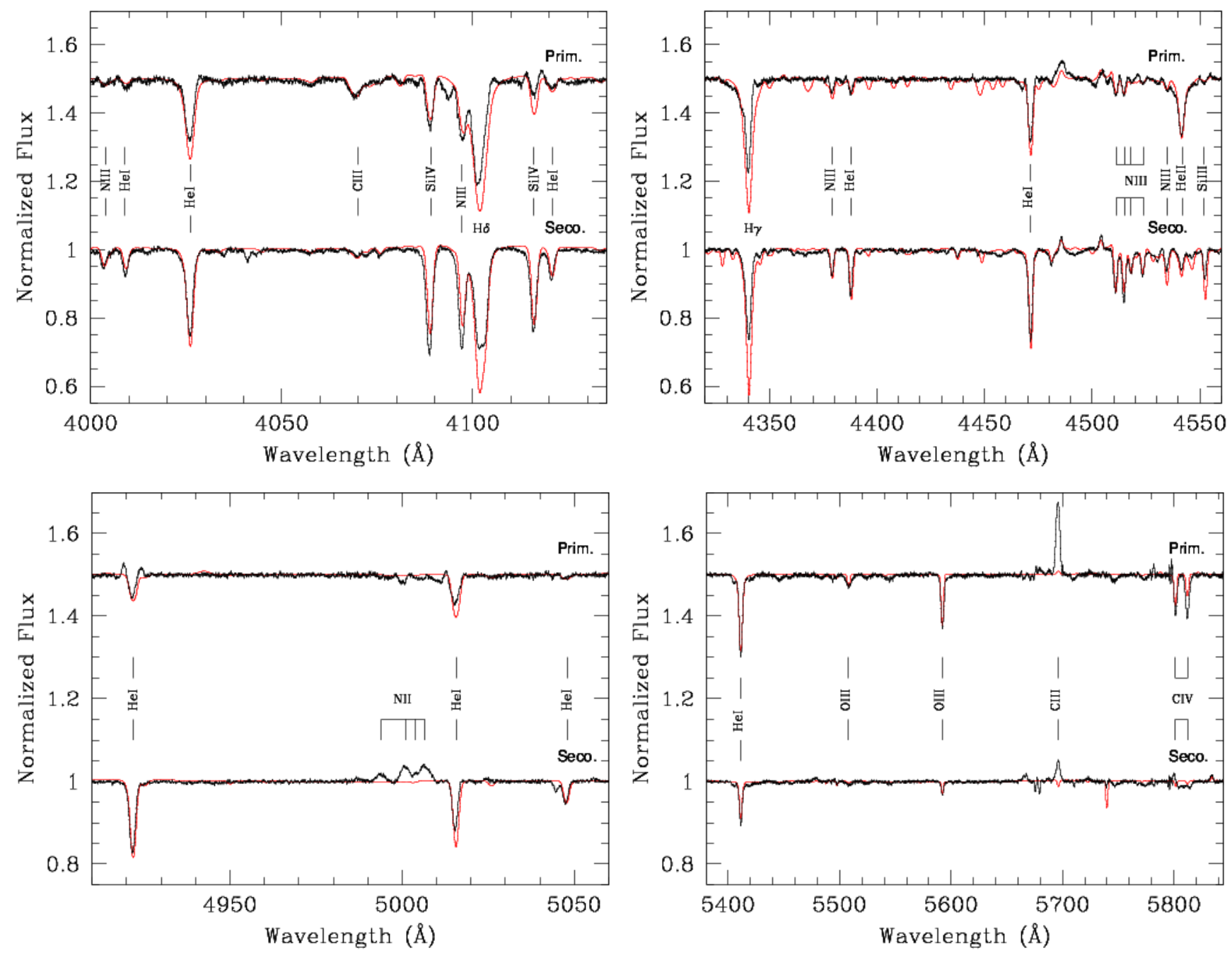

Fig. 1. Part of the normalized separated spectra of the primary (top, shifted upwards by 0.5 continuum units) and secondary stars (bottom), along with the best-fit CMFGEN model spectra (red).

After the fundamental stellar parameters were established or fixed, we investigated the CNO abundances through the strengths of the associated lines. We performed a normalized $\chi^{2}$ analysis to determine the best fit to selected lines (Martins et al. 2015). The normalization consists in dividing the $\chi^{2}$ by its value at minimum, $\chi^{2}{ }_{\min }$. As a $1 \sigma$ uncertainty on the abundances, we then considered abundances up to a $\chi^{2}$ of 2.0 , that is, an approximation for 1 over $\chi^{2}{ }_{\text {min }}$, as suggested by Martins et al. (2015). We used the C III $\lambda \lambda$ 4068-70, C II $\lambda$ 4267, C III $\lambda 5826$, N III $\lambda 4004$, N III $\lambda \lambda 4511-15-18-24$, N II $\lambda 5026$, O III $\lambda 5508$, and $\mathrm{O}$ III $\lambda 5592$ lines to adjust the $\mathrm{C}, \mathrm{N}$, and $\mathrm{O}$ abundances of the primary star. For the secondary star, the same lines were used for the $\mathrm{N}$ and $\mathrm{O}$ abundances, but the only line used for the $\mathrm{C}$ abundance was C III $\lambda \lambda$ 4068-70. Two methods were used to determine the $\chi^{2}$ of each line. First, we compared the depth of the observed and synthetic line cores in the calculation. Second, we determined the mean $\chi^{2}$ for every independent point of the full profile of each considered line. The two methods gave similar results. After testing the influence of the errors on projected rotational velocities on the determination of the surface abundances, we found that their effect was small compared to the $1 \sigma$ uncertainties on the measured abundances as determined here above. We therefore neglected them.

\subsection{Results}

Figure 1 illustrates the best fit of the optical spectra of the primary and secondary stars obtained with CMFGEN. In Table 4 we present the stellar parameters determined compared to those derived by Rauw et al. (2001). In general, these two works agree well. Table 5 compares the chemical abundances of these best-fit models with solar abundances taken from Asplund et al. (2009).

Figure 1 shows that the He lines and the C III $\lambda \lambda$ 4068-70 lines are very well reproduced for both stars. The C III $\lambda 5696$ line cannot be considered in the determination of the surface $\mathrm{C}$ abundance since its formation process depends on a number of fine details of atomic physics and of the modelling (Martins $\&$ Hillier 2012). Most of the $\mathrm{N}$ lines also are very well adjusted. The N II emission lines around $5000 \AA$ in the primary spectrum may be due to emission in the interaction zone, as they are well present in the observed spectrum and not represented in the modelled spectrum. The case of oxygen is more problematic. Whilst O III $\lambda 5592$ is well adjusted, several other O III lines are present in the synthetic CMFGEN spectra (e.g. O III $\lambda \lambda$ 4368, 4396, 4448,4454 , and 4458), but they are barely visible in the separated spectra. We checked that these lines are also absent from the original observed binary spectra. Their absence is thus by no means an artefact that is due to the treatment of our data. 
Table 4. Stellar parameters of the primary and secondary stars as obtained with CMFGEN.

\begin{tabular}{l|cccc}
\hline \hline & \multicolumn{2}{c}{ This study } & \multicolumn{2}{c}{ Rauw et al. (2001) } \\
& Prim. & Sec. & Prim. & Sec. \\
\hline$R\left(R_{\odot}\right)$ & $19.3 \pm 2.2$ & $25.9 \pm 3.4$ & $24.3 \pm 0.7$ & $28.1 \pm 0.7$ \\
$M\left(M_{\odot}\right)$ & $50.5 \pm 20.1$ & $31.9 \pm 9.5$ & $54.8 \pm 4.6$ & $33.0 \pm 2.8$ \\
$T_{\text {eff }}\left(10^{4} \mathrm{~K}\right)$ & $3.40 \pm 0.15$ & $2.80 \pm 0.15$ & $3.51 \pm 0.1$ & $3.05 \pm 0.04$ \\
$\log \left(\frac{L}{L_{\odot}}\right)$ & $5.63 \pm 0.05$ & $5.58 \pm 0.04$ & $5.90 \pm 0.08$ & $5.78 \pm 0.08$ \\
$\log g(\mathrm{cgs})$ & $3.55 \pm 0.15$ & $3.05 \pm 0.15$ & & \\
$\beta$ & $1.03^{f}$ & $1.08^{f}$ & & \\
$v_{\infty}\left(\mathrm{km} \mathrm{s}^{-1}\right)$ & $2450^{f}$ & $2450^{f}$ & & \\
$\dot{M}\left(M_{\odot} \mathrm{yr}^{-1}\right)$ & $9.2 \times 10^{-7 f}$ & $3.3 \times 10^{-7 f}$ & & \\
$\mathrm{BC}$ & -3.17 & -2.67 & & \\
\hline
\end{tabular}

Notes. The best-fit CMFGEN model parameters are compared with the parameters obtained by Rauw et al. (2001) for an orbital inclination of $21^{\circ}$. The effective temperatures from Rauw et al. (2001) were derived through the effective temperature calibration of Chlebowski \& Garmany (1991) and permitted, along with the determined luminosities, inferring the stellar radii. The quoted errors correspond to $1 \sigma$ uncertainties. The symbol ${ }^{(f)}$ in the table corresponds to values fixed from the literature (Howarth et al. 1997; Muijres et al. 2012). The bolometric corrections are taken from Lanz \& Hubeny (2003), based on our best-fit $T_{\text {eff }}$ and $\log g$.

Table 5. Chemical abundances of the components of HD 149404.

\begin{tabular}{l|ccc}
\hline \hline & Primary & Secondary & Sun \\
\hline $\mathrm{He} / \mathrm{H}$ & 0.1 & 0.1 & 0.089 \\
$\mathrm{C} / \mathrm{H}$ & $1.02_{-0.11}^{+0.10} \times 10^{-4}$ & $1.89_{-0.47}^{+0.47} \times 10^{-5}$ & $2.69 \times 10^{-4}$ \\
$\mathrm{~N} / \mathrm{H}$ & $1.32_{-0.15}^{+0.20} \times 10^{-4}$ & $7.15_{-1.8}^{+2.5} \times 10^{-4}$ & $6.76 \times 10^{-5}$ \\
$\mathrm{O} / \mathrm{H}$ & $7.33_{-1.1}^{+1.1} \times 10^{-4}$ & $7.85_{-1.1}^{+1.8} \times 10^{-5}$ & $4.90 \times 10^{-4}$ \\
\hline
\end{tabular}

Notes. Abundances are given by number as obtained with CMFGEN. The solar abundances (Asplund et al. 2009) are quoted in the last column. The $1 \sigma$ uncertainty on the abundances was set to abundances corresponding to a $\chi^{2}$ of 2.0. As only one line was considered for the $\mathrm{C}$ abundance of the secondary, the corresponding calculated uncertainty was unrealistically low. A similar problem was encountered for the $\mathrm{O}$ abundance of the primary star, for which we only had two lines to work with and several absent lines over the spectrum, as specified in the second paragraph of this section. To circumvent these problems, we set the uncertainties for these two abundances to values close to those calculated for the other two elements of the corresponding star.

We stress two interesting results from our spectral analysis. First of all, we confirm the large overabundance of $\mathrm{N}$ in the secondary spectrum. Second, we infer an asynchronous rotation of the two stars. The radii determined with CMFGEN (Table 4), the mean projected rotational velocities (Table 1), and the inclination estimate from Rauw et al. (2001) yield the rotation periods of the primary and secondary stars: $3.77 \pm 0.32$ days and $7.46 \pm 0.95$ days, respectively, or a period ratio of $0.50 \pm 0.11$. We note that within the uncertainties on the inclination, the secondary rotational period is similar to the orbital period of the system (9.81 days).We return to these points in Sect. 5. Finally, we note that according to the radii determined here, none of the stars currently fills its Roche lobe. With the formula of Eggleton (1983), we estimate a Roche-lobe volume filling factor of $15 \%$ for the primary and $73 \%$ for the secondary.

As pointed out above, disentangling the IUE spectra of HD 149404 was more problematic, especially for those parts of the UV spectra that feature strong and broad wind lines and/or are polluted by blends with numerous interstellar features. The left panel of Fig. 2 illustrates the comparison of the separated spectra and the synthetic spectra obtained with CMFGEN over a wavelength range that is relatively free of interstellar lines and mainly hosts photospheric lines. The agreement is clearly quite reasonable.
To check whether our CMFGEN models are also able to correctly reproduce the wind features, we re-combined the synthetic CMFGEN UV spectra of the primary and secondary taking into account the radial velocity shifts at the time of the observations. We did so for several orbital phases. The right panel of Fig. 2 illustrates the result for three selected IUE spectra (SWP03008, JD 2443 798.965, SWP02755, JD 2443 776.869, and SWP54350, JD 2449817.595$)$, corresponding to conjunction phase 0.00 and quadrature phases 0.75 and 0.25 . The synthetic spectrum clearly agrees well with the observation, except for a few narrow absorptions that are due to the interstellar medium, which are not taken into account in our model. This additionally supports our determination of the physical parameters of the system components. Moreover, the concordance between the synthetic and observed UV spectra suggests that the P-Cygni profiles form over parts of the stellar winds that are not significantly affected by the wind-wind interactions, the latter leading to a loss of spherical symmetry of the line formation region.

The fluxed CMFGEN spectra also allow us to estimate the brightness ratio (primary/secondary) in the UV. We find a value of $0.91^{2}$.

Furthermore, we found relatively little variability of the P-Cygni profiles over the orbital period. Both observations (lack of strong variability and little effect of wind interaction on the line morphology) are likely due to the low orbital inclination $\left(i \sim 21^{\circ}\right)$ of the system. This inclination implies that the observed spectrum arises mostly at high stellar latitudes, where the wind-wind interaction zone has less influence on the wind structure.

\section{Discussion and conclusion}

\subsection{Evolutionary status}

As we have shown in the previous section, the spectra of the components of HD 149404 display the signatures of some

\footnotetext{
2 This result is at odds with the $0.7 \mathrm{mag}$ difference (i.e. primary/secondary brightness ratio of 1.91) between the primary and secondary UV fluxes inferred by Howarth et al. (1997). This situation most likely stems from the differences in the spectral types adopted by Howarth et al. (1997), who classified the system as O8.5 I + O7 III, whilst we have derived O7.5 If + ON9.7 I. This difference in spectral types directly affects the strength of the cross-correlation peaks of the spectra of the two components with the template spectrum of $\tau$ Sco (B0.2 IV) and thus the correction on the magnitude difference inferred by Howarth et al. (1997).
} 

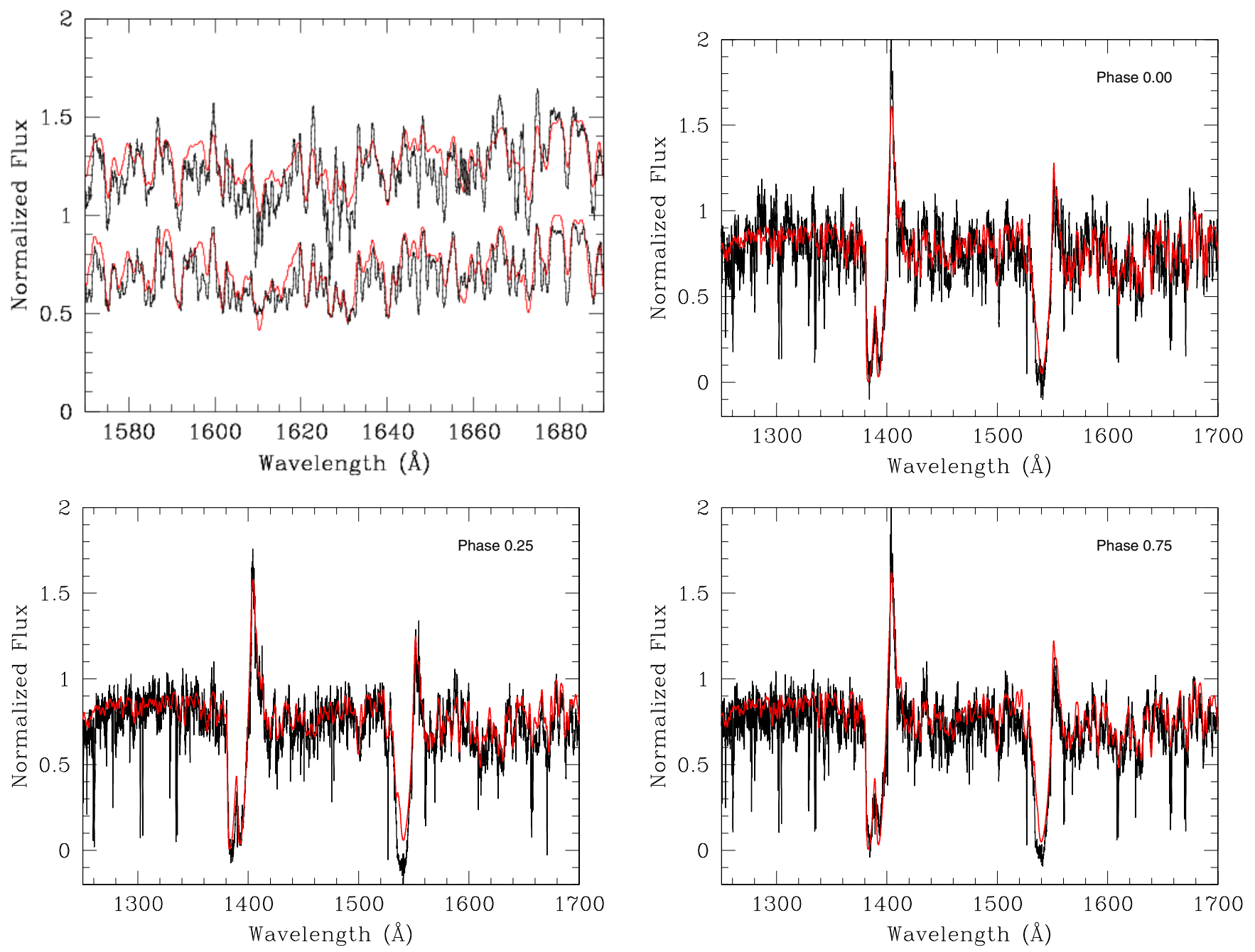

Fig. 2. Upper left panel: comparison between the separated IUE spectra (black) of the primary and secondary stars around the He II $\lambda 1640$ line and the corresponding synthetic spectra (red). The primary spectrum is shifted upwards by 0.5 for clarity. The other three panels display a comparison between the SWP03008 (upper right), SWP54350 (lower left) and SWP02755 (lower right) IUE spectra taken at phases $0.0,0.25$ and 0.75 in black and the synthetic binary spectra obtained through combination of CMFGEN primary and secondary spectra at given phases in red.

enrichment by the products of stellar nucleosynthesis processes. These properties indicate a previous mass-exchange episode. Figure 3 compares our inferred N/C and N/O ratios with the predictions for the evolution of single massive stars (Ekström et al. 2012) either without rotation (left panel) or with a rotational velocity of $0.4 \times v_{\text {crit }}$ (right panel). This figure clearly shows that for the spectroscopic masses of the primary and secondary stars these models cannot account for the observed CNO abundance patterns. For instance, in the case of models without rotation (left-hand panel of Fig. 3), no chemical enrichment is expected during the main-sequence evolution for stars with mass $<60 M_{\odot}$. Only for the $M>60 M_{\odot}$ model is the loss of material through stellar wind sufficient to reveal the products of the CNO cycle at the surface before the end of the main-sequence evolution. Adopting instead the rotating models (right-hand panel of Fig. 3), the enrichment of the secondary can only be explained by single-star evolution if we assume at least a star with $60 M_{\odot}$, whilst the spectroscopic mass is about a factor two lower. When the surface abundances of a single star of $60 M_{\odot}$ initial mass reach the values observed for the secondary of HD 149404, the current mass of the star is still about $50 M_{\odot}$, that is, it is much higher than the mass determined for the secondary star.

The observed CNO abundance pattern is qualitatively much easier to interpret if the present-day secondary star was formerly the more massive component of the binary and transferred mass and angular momentum to the present-day primary in an RLOF episode. During this process, the outer envelope of the secondary was removed, revealing layers of material that were previously inside the convective hydrogen-burning core (Vanbeveren 1982, 2011).

We start by comparing our results with theoretical predictions from the literature. The expectation is that the more mass has been lost by the donor, the deeper the layers that are revealed at the surface and thus the higher the $\mathrm{N} / \mathrm{C}$ ratio. The predicted $\mathrm{CNO}$ abundances for the mass loser after a case B mass-transfer episode are $[\mathrm{N} / \mathrm{C}]=100[\mathrm{~N} / \mathrm{C}]_{\odot}$ and $[\mathrm{N} / \mathrm{O}] \geq 5[\mathrm{~N} / \mathrm{O}]_{\odot}$ (Vanbeveren 1982). This is indeed close to what we observe in our analysis. Moreover, the RLOF scenario is re-enforced by the values of the primary star abundances. Classical radiative equilibrium evolutionary models predict normal $\mathrm{H}$ and $\mathrm{He}$ abundances for the mass gainer of an RLOF episode, but an $\mathrm{N}$ abundance of two to three times solar (Vanbeveren \& de Loore 1994), which agrees with our results.

There are some caveats here, however. First, the results of Vanbeveren (1982) apply to case B mass transfer, whereas HD 149404 is more likely to have undergone a case A mass exchange (see below). Second, when the outer hydrogen-rich layers are removed, an enrichment of the stellar surface of the mass 

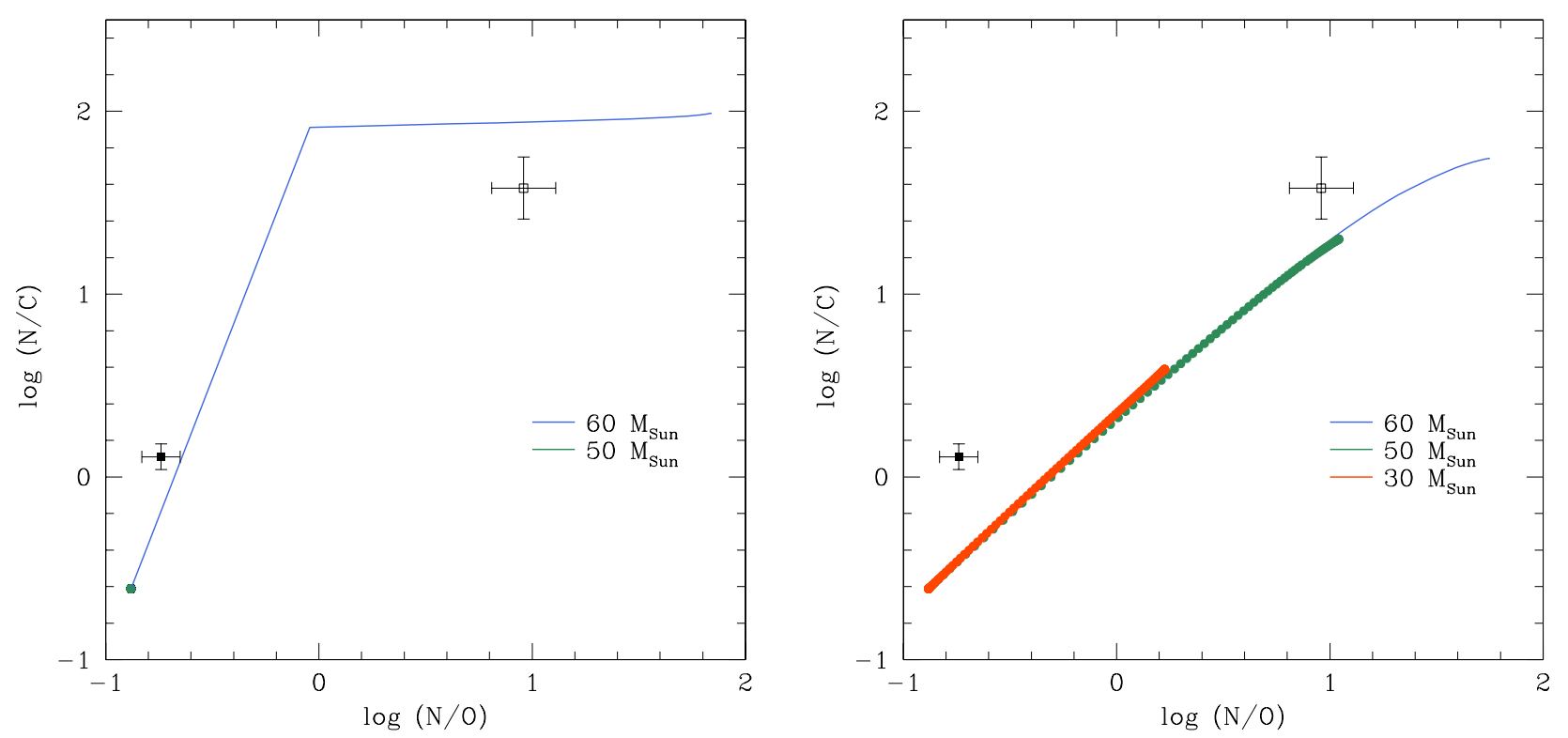

Fig. 3. Comparison of the N/C and N/O ratios determined from our spectral analyses with predictions from single-star evolutionary models of different masses (Ekström et al. 2012). The primary and secondary stars are shown by the open and filled square symbols, respectively. The left panel illustrates the results for core hydrogen-burning phase tracks without stellar rotation, whilst the right panel corresponds to the same tracks for stars rotating at $0.4 \times v_{\text {crit }}$.

donor in helium is expected. Our spectral modelling was made with the He abundance set to solar, however, and there is no hint for our models requiring a He enhancement in any of the stars. Although the predicted relative change of helium surface abundance is usually much smaller than for $\mathrm{N}$, this result is nonetheless surprising, especially in view of the large $\mathrm{N}$ overabundance of the secondary star.

To illustrate the helium enrichment that might be expected, we consider once more the single-star evolutionary tracks with rotation of Ekström et al. (2012). In these models, a nitrogen surface abundance similar to the one observed for the secondary is always accompanied by an increase of the surface helium abundance to values higher than $y=0.35$ by mass. The fact that the spectrum of the secondary of HD 149404 can be fit with a solar helium abundance $(y=0.1)$ suggests that the internal structure of the secondary before the onset of mass transfer must have been different from that expected from single-star evolutionary models. Moreover, the RLOF in HD 149404 must have come to a (temporary) stop before the full hydrogen-rich envelope of the mass donor was removed. The latter conclusion is also supported by our finding that both stars are currently well inside their Roche lobes.

Given the present-day spectroscopic masses, the secondary must have lost at least $9.3 M_{\odot}$ assuming a fully conservative mass transfer. Conservative RLOF will increase the period of the binary. If we assume a fully conservative mass transfer and apply the formula of Vanbeveren et al. (1998), we find that the orbital period would have increased by $17 \%$ at most, the shortest possible initial orbital period being 8.4 days for a mass exchange of $9.3 M_{\odot}$. However, Petrovic et al. (2005) argued that in fast case A RLOF, only about $15-20 \%$ of the mass shed by the mass donor is indeed accreted by its companion. If the mass-transfer efficiency is that low, then the secondary must have lost a much larger amount of mass. Such a non-conservative mass transfer can result from the transfer of angular momentum. In fact, the mass gainer can be spun up to critical rotation and will then repel additional mass (and angular momentum), leading to a nonconservative mass transfer (e.g. Langer et al. 2003; Vanbeveren 2011; Langer 2012). In the previous section, we noted that the components of the system are apparently not in synchronous rotation. This asynchronicity therefore also suggests the existence of a past mass and angular momentum transfer episode, with a clear spin up of the primary star.

In Fig. 4 we present the positions of the primary and secondary stars in the Hertzsprung-Russell diagram (HRD) (upper panels) and in the $\log g-\log T_{\text {eff }}$ diagram (lower panels), along with the evolutionary tracks from Ekström et al. (2012) for the core-hydrogen burning phase of single stars at solar metallicity, both for non-rotating (left panels) and rotating (right panels) stars. This figure again illustrates the failure of single-star evolutionary tracks to account for the properties of the components of HD 149404. If we consider the models without rotational mixing, the position of the primary star in the HRD is relatively close to the track corresponding to its present-day spectroscopic mass. However, the secondary appears too luminous for its present-day mass compared to these evolutionary tracks. If we compare the stars to the $40 \%$ critical rotation case, we find that the primary's luminosity is below that expected for its present-day spectroscopic mass. Moreover, the secondary lies outside the range covered by tracks in the core-hydrogen burning phase. If we consider the non-rotating case, we see that in the $\log g-\log T_{\text {eff }}$ diagram the primary star has a lower effective temperature and a higher surface gravity than expected for a $50 M_{\odot}$ star, whilst the secondary star displays parameters corresponding to stars twice as massive as its present-day spectroscopic mass. For stars rotating at $40 \%$ of their critical velocity, we find that both stars would have initial masses of about $30 M_{\odot}$. The primary dynamical mass is about a factor 2 higher, while for the secondary the agreement is good. Finally, in all cases (with and without rotation and for both diagrams), the two components of HD 149404 are not located on the same isochrone, although they would be expected to have the same age ${ }^{3}$.

In a mass-transfer episode, the location of both stars in the HRD after the RLOF strongly depends on the details of the process. For instance, spinning-up the mass gainer can lead to

3 The age of the Ara OB1 association of which HD 149404 is a member was estimated in the literature as $~ 3 \mathrm{Myr}$ (Wolk et al. 2008). 
F. Raucq et al.: Signatures of past mass exchange in HD 149404
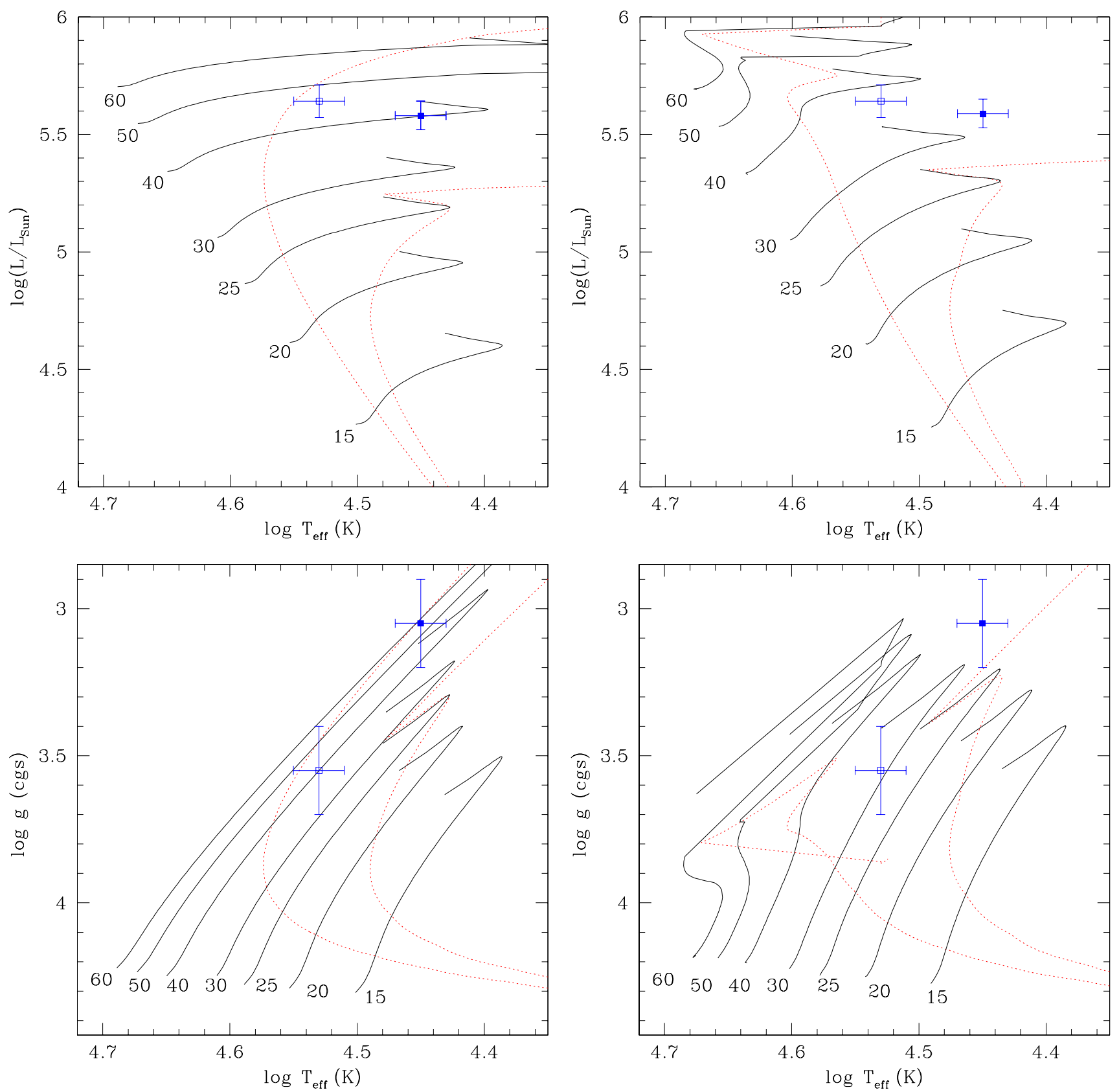

Fig. 4. Position of the primary (open square) and secondary (filled square) stars in the Hertzsprung-Russell diagram (upper panels) and the log $g$ $\log T_{\text {eff }}$ diagram (lower panels) along with evolutionary tracks for single massive stars at solar metallicity during the core-hydrogen burning phase (Ekström et al. 2012). In the left panels, the evolutionary tracks correspond to non-rotating stars, whilst the right panels yield the results for stars rotating at $0.4 \times v_{\text {crit }}$. The dotted red lines correspond to isochrones of 3.2 and 6.3 million years for the left panels and of 4.0 and 8.0 million years for the right panels.

almost complete mixing of the latter. In this situation, models predict that He would be enhanced in the atmosphere of the mass gainer and that the latter would become bluer and overluminous for its mass (Vanbeveren \& de Loore 1994). If there is no strong spin-up and the accretion occurs rather slowly and is accompanied by a fast diffusion process, then the location of the gainer in the HRD should be very close to that of a normal star of the same mass and chemical composition (Vanbeveren et al. 1998). The star is rejuvenated, however: it lies on an isochrone that corresponds to a younger age than its actual age. The location of the primary in the HRD suggests that in the present case the second scenario is likely to apply with a very moderate rejuvenation, if any, of the primary. This conclusion is supported by the lack of a strong He enrichment, but it is at odds with the highly nonsynchronous rotation of the primary reported above.

\subsection{Comparison with other post-RLOF systems}

In this section, we briefly compare the properties of HD 149404 with those of two other O-type binaries, Plaskett's Star and LZCephei, which are probably in a post-case A RLOF evolutionary stage and have been analysed in a way similar to the present work (Linder et al. 2008; Mahy et al. 2011).

Plaskett's Star (HD 47 129) is an O8 III/I + O7.5 III binary with an orbital period of 14.4 days, slightly longer than that of HD 149404, and a mass ratio near unity. Two observational 
facts suggest that the initially more massive primary has undergone a case A RLOF episode in the past (Linder et al. 2008). First, the projected rotational velocity of the secondary star is remarkably high (mean value of $272.5 \pm 35.0 \mathrm{~km} \mathrm{~s}^{-1}$ ) compared to that of the primary (mean value of $65.7 \pm 6.3 \mathrm{~km} \mathrm{~s}^{-1}$ ). This strongly asynchronous rotation likely stems from a spin-up of the secondary star through a recent transfer of angular momentum from the primary star. Second, the primary star displays nitrogen overabundance $\left(\mathrm{N} / \mathrm{N}_{\odot}=16.6 \pm 5.0\right)$ and carbon depletion $\left(\mathrm{C} / \mathrm{C}_{\odot}=0.2 \pm 0.1\right)$, as is typical of an advanced evolutionary stage, whilst no such nitrogen enrichment is seen in the secondary spectrum ${ }^{4}$. Given the present-day configuration of HD 47129 , it seems that the transfer of angular momentum and the resulting spin-up of the mass gainer (the secondary in this case) might have prevented accretion of a larger portion of the primary's mass.

LZ Cephei (HD 209481) is an O9 III + ON9.7 V binary with a short orbital period of 3.07 days (Kameswara Rao 1972) and a mass ratio (primary/secondary) of 2.53 (Mahy et al. 2011). Both components of LZ Cep almost fill their Roche lobe (Mahy et al. 2011; Palate et al. 2013). Mahy et al. (2011) found that the secondary displays a strong nitrogen enrichment (18 times solar), carbon and oxygen depletion ( 0.1 times solar), and a high helium content (4 times solar), suggesting a very advanced state of evolution. On the other hand, the primary displays only slightly altered surface abundances consistent with single-star evolution. This suggests that the mass transfer was strongly nonconservative. Mahy et al. (2011) noted a slight asynchronicity of the rotation periods of the order of $10-20 \%$, but concluded that the system is probably on the verge of achieving synchronization. Comparison with single-star evolutionary tracks indicates a similar age discrepancy as we have found here for HD 149404. The evolutionary masses $\left(25.3_{-4.0}^{+6.2}\right.$ and $18.0_{-2.5}^{+2.4} M_{\odot}$, for the primary and secondary respectively) are significantly higher than their dynamical masses $\left(16.9 \pm 1.0\right.$ and $6.7 \pm 1.0 M_{\odot}$ respectively, Mahy et al. 2011; Palate et al. 2013).

Although our sample is currently too limited to draw firm conclusions, the above comparison reveals several interesting results that are worth studying in more detail with a larger sample. First, we note that in all three systems, the components appear either over- or under-luminous when compared with single-star evolutionary tracks corresponding to their present-day masses. During its initial phases, case A mass transfer occurs on a thermal timescale leading to a very rapid mass exchange. However, the mass transfer subsequently slows down and occurs on a nuclear timescale instead. By the time the mass transfer ends, both stars would therefore be expected to be in thermal equilibrium again. Since all three systems under consideration are in a detached configuration, the stars should thus have settled onto their new post-RLOF evolutionary tracks. The discrepancy between the observed luminosities and those predicted by the single-star evolutionary tracks most likely stems from several effects, such as observational uncertainties, altered chemical compositions (and hence opacities of the stellar material), and a rotational velocity history that differs from that assumed in the single-star models.

Our comparison shows that the degree of asynchronicity in the three post-RLOF systems increases with orbital period (from

\footnotetext{
4 The apparent $\mathrm{N}$ underabundance of the secondary inferred by Linder et al. (2008) could be an artefact that is due to the relatively shallow nitrogen lines of the secondary being washed out by its fast rotation and the difficulties of properly normalizing echelle spectra with such broad and shallow features (see also Fig. 1 of Palate \& Rauw 2014).
}

LZCep, over HD 149404 to HD 47 129). This is most probably due to the higher efficiency of the tidal forcing in shorter period systems, or it might be seen as evidence that momentum transfer has played a larger role in those systems that have a wider present-day orbital separation. This is expected since binary evolution models predict spin-up to be less efficient in closer systems because in such systems the accretion stream directly affects the mass gainer without forming an accretion disk (e.g. Langer 2012). The questions of synchronization and circularization in close binary systems with early-type stars, featuring radiative envelopes and convective cores, have been addressed by Zahn (1975) and Tassoul (1987). Zahn (1975) considered radiative damping of the dynamical tide in the outer layers of the stellar envelopes as the main mechanism for synchronization. The corresponding synchronization timescales strongly depend upon the tidal torque constant $E_{2}$, which varies by several orders of magnitude during the main-sequence lifetime of the star (Zahn 1977). Siess et al. (2013) provided a parametrization of this parameter as a function of stellar mass and relative age of the star. Assuming that their formalism holds for the masses of the stars considered here and adopting an age of half the mainsequence age of the stars, we estimated the values of $E_{2}$. We then evaluated the synchronization times following Eq. (4.28) of Zahn (1975) by means of moments of inertia tabulated by Claret \& Giménez (1992). The results are $5.6 \times 10^{5}$ and $1.4 \times 10^{4}$ years for the primary and secondary stars of HD 149404, respectively, $4.4 \times 10^{6}$ and $27.6 \times 10^{6}$ years for the primary and secondary of Plaskett's star, and $2.8 \times 10^{4}$ and $2.5 \times 10^{4}$ years for the primary and secondary components of LZ Cephei. Tassoul (1987), on the other hand, proposed that the most effective mechanism for synchronization is provided by large-scale hydrodynamic motions in the interiors of the tidally distorted binary components. The resulting timescales are considerably shorter than those estimated following the formalism of Zahn (1975). We estimate synchronization timescales of $2.1 \times 10^{4}$ and $3.2 \times 10^{3}$ years for HD $149404,7.2 \times 10^{4}$ and $2.0 \times 10^{5}$ years for Plaskett's star, and $1.6 \times 10^{3}$ and $6 \times 10^{2}$ years for LZ Cep. Regardless of which of the two formalisms is most appropriate to describe the evolution of rotation in the systems considered here, we find that, except perhaps for Plaskett's Star, which has a longer synchronization timescale, the RLOF phase probably must have ended less than a few $10^{4}$ years ago.

Another puzzling result is the fact that the post-RLOF massratio deviates most from unity for the shortest period system (LZ Cep). Therefore, in this system, the RLOF must have been comparatively more efficient than in the longer period systems. As discussed above, the mass transfer in LZCep was most likely highly non-conservative. If the material lost by the system does not carry away angular momentum in addition to the intrinsic orbital angular momentum, such a non-conservative mass loss leads to a widening of the orbital separation $a$ given by

$$
\frac{\dot{a}}{a}=-\frac{\dot{M}}{M}
$$

where $\dot{M}<0$ is the mass-loss rate (Singh \& Chaubey 1986; Tout $\&$ Hall 1991). Assuming a fully non-conservative mass loss, this would imply that the initial orbital period of LZ Cep must have been shorter than 1.5 days. The duration of the RLOF episode depends on the evolution of the Roche-lobe filling factor of the mass donor. The evolution of the size of the Roche lobe is set by the relative importance of the orbit expansion and the shrinking of the relative size of the mass donor's Roche lobe. In addition, the evolution of the radius of the mass donor needs to be accounted for (see Tout \& Hall 1991). Since massive stars 
with radiative envelopes contract considerably on a dynamical timescale (Tout \& Hall 1991) and in view of the current massratio of LZ Cep (Mahy et al. 2011), it seems unlikely that a nonconservative RLOF could be the explanation for a more efficient mass loss. Alternatively, if the material lost by the system carried off additional angular momentum, then the result could be a shrinking of the orbit. In active, low-mass stars, such a situation could arise if the material lost by the donor corotates with the latter under the effect of a magnetic field out to a wide distance (Tout \& Hall 1991). However, strong magnetic fields are fairly rare among single massive stars $(\sim 7 \%)$, and their incidence appears even lower among massive binaries (Neiner et al. 2015). Hence, this scenario also appears very unlikely in the case of LZ Cep.

Clearly, the above trends call for confirmation by studying a larger sample of post-case A RLOF massive binary systems. We are currently undertaking such studies, and the results will be presented in forthcoming publications.

\subsection{Summary and conclusion}

We analysed the fundamental properties of the binary system HD 149404 by means of spectral disentangling and the CMFGEN atmosphere code. Our investigation of this object represents a significant improvement over previous studies because we have obtained the individual spectra of each component. This led to the determination of a number of stellar parameters that were used to constrain the evolutionary status of the system.

We established the existence of a large overabundance in $\mathrm{N}$ and a $\mathrm{C}$ and $\mathrm{O}$ depletion in the secondary star and also found a slight $\mathrm{N}$ enhancement in the primary's spectrum. We showed that these surface abundances cannot be explained by single-star evolutionary models. Furthermore, we inferred an asynchronous rotation of the two stars of the system. These two results indicate a previous mass and angular momentum-exchange phase through a Roche-lobe overflow episode. Given the present status of HD 149404 as an O+O binary, this mass transfer probably was a case A RLOF. However, dedicated theoretical studies are needed to understand the details of the evolution of this system.

Acknowledgements. The Liège team acknowledges support from the Fonds de Recherche Scientifique (FRS/FNRS) including especially an FRS/FNRS Research Project (T.0100.15), as well as through an ARC grant for Concerted Research Actions financed by the French Community of Belgium (WalloniaBrussels Federation), and an XMM PRODEX contract (Belspo). Anthony Hervé is supported by grant 14-02385S from GA ČR.

\section{References}

Asplund, M., Grevesse, N., Sauval, A. J., \& Scott, P. 2009, ARA\&A, 47, 481 Chlebowski, T., \& Garmany, C. D. 1991, ApJ, 368, 241

Claret, A., \& Giménez, A. 1992, A\&AS, 96, 255

Conti, P. S. 1973, ApJ, 179, 161

Conti, P. S. 1974, ApJ, 187, 539

Conti, P. S., \& Alschuler, W. R. 1971, ApJ, 170, 325

Conti, P. S., \& Frost, S. A. 1977, ApJ, 212, 728 de Loore, C., \& Vanbeveren, D. 1994, A\&A, 292, 463

de Mink, S. E., Pols, O. R., \& Hilditch, R. W. 2007, A\&A, 467, 1181

de Mink, S. E., Cantiello, M., Langer, N., et al. 2009, A\&A, 497, 243

Dray, L. M., \& Tout, C. A. 2007, MNRAS, 376, 61

Eggleton, P. P. 1983, ApJ, 268, 368

Ekström, S., Georgy, C., Eggenberger, P., et al. 2012, A\&A, 537, A146

González, J. F., \& Levato, H. 2006, A\&A, 448, 283

Gray, D. F. 2008, The Observation and Analysis of Stellar Photospheres, 3rd edn. (Cambridge University Press)

Gray, R. O. 2010, http://www.appstate.edu/ grayro/spectrum/ spectrum276/node38.html

Herbst, W., \& Havlen, R. J. 1977, A\&AS, 30, 279

Hillier, D. J., \& Miller, D. L. 1998, ApJ, 496, 407

Howarth, I. D., Siebert, K. W., Hussain, G. A. J., \& Prinja, R. K. 1997, MNRAS, 284,265

Hurley, J. R., Tout, C. A., \& Pols, O. R. 2002, MNRAS, 329, 897

Kameswara Rao, N. 1972, PASP, 84, 563

Kippenhahn, R., \& Weigert, A. 1967, Z. Astrophys., 66, 58

Lanz, T., \& Hubeny, I. 2003, ApJS, 146, 417

Langer, N. 2012, ARA\&A, 50, 107

Langer, N., Wellstein, S., \& Petrovic, J. 2003, Proc. IAU Symp., 212, 275

Linder, N., Rauw, G., Martins, F., et al. 2008, A\&A, 489, 713

Mahy, L., Martins, F., Machado, C., et al. 2011, A\&A, 533, A9

Mahy, L., Gosset, E., Sana, H., et al. 2012, A\&A, 540, A97

Martins, F. 2011, Bull. Soc. Roy. Sci. Liège, 80, 29

Martins, F., \& Hillier, D. J. 2012, A\&A, 545, A95

Martins, F., \& Plez, B. 2006, A\&A, 457, 637

Martins, F., Schaerer, D., \& Hillier, D. J. 2005, A\&A, 436, 1049

Martins, F., Hervé, A., Bouret, J.-C., et al. 2015, A\&A, 575, A34

Muijres, L. E., Vink, J. S., de Koter, A., Müller, P. E., \& Langer, N. 2012, A\&A, 537, A37

Nazé, Y., Carrier, F., \& Rauw, G. 2002, in Interacting Winds from Massive Stars, eds. A. F. J. Moffat, \& N. St-Louis, ASP Conf. Proc., 260, 457

Neiner, C., Mathis, S., Alecian, E., et al. 2015, in Proc. IAU, 305, 61

Palate, M., \& Rauw, G. 2014, A\&A, 572, A16

Palate, M., Rauw, G., \& Mahy, L. 2013, Central European Astrophysical Bulletin, 37, 311

Petrovic, J., Langer, N., \& van der Hucht, K. A. 2005, A\&A, 435, 1013

Podsiadlowski, P., Joss, P. C., \& Hsu, J. J. L. 1992, ApJ, 391, 246

Rauw, G. 2007, DSc Thesis, University of Liège, Belgium

Rauw, G. 2013, in Setting a New Standard in the Analysis of Binary Stars, eds.

K. Pavlovski, A. Tkachenko, \& G. Torres, EAS Pub. Ser., 64, 59

Rauw, G., Nazé, Y., Carrier, F., et al. 2001, A\&A, 368, 212

Reed, B. C. 2003, AJ, 125, 2531

Sana, H., de Mink, S. E., de Koter, A., et al. 2012, Science, 337, 444

Siess, L., Izzard, R. G., Davis, P. J., \& Deschamps, R. 2013, A\&A, 550, A100

Simón-Díaz, S., \& Herrero, A. 2007, A\&A, 468, 1063

Simón-Díaz, S., \& Herrero, A. 2014, A\&A, 562, A135

Singh, M., \& Chaubey, U. S. 1986, Ap\&SS, 124, 389

Stickland, D. J., \& Koch, R. H. 1996, The Observatory, 116, 145

Tassoul, J.-L. 1987, ApJ, 322, 856

Thaller, M. L., Gies, D. R., Fullerton, A. W., Kaper, L., \& Wiemker, R. 2001, ApJ, 554, 1070

Tout, C. A., \& Hall, D. S. 1991, MNRAS, 253, 9

Vanbeveren, D. 1982, A\&A, 105, 260

Vanbeveren, D. 2011, Bull. Soc. Roy. Sci. Liège, 80, 530

Vanbeveren, D., \& de Loore, C. 1994, A\&A, 290, 129

Vanbeveren, D., de Loore, C., Van Rensbergen, W. 1998, A\&ARv, 9, 63

van der Hucht, K. A. 1996, in Wolf-Rayet stars in the framework of stellar evolution, Liège Int. Astrophys. Colloq., 33, eds. J.-M. Vreux, A. Detal, D. Fraipont-Caro, E. Gosset, \& G. Rauw, 1

Wellstein, S., Langer, N., \& Braun, H. 2001, A\&A, 369, 939

Wolk, S. J., Comerón, F., \& Bourke, T. 2008, Handbook of Star Forming Regions, Vol. II: The Southern Sky, ASP Monograph Publications, Vol. 5. ed. Bo Reipurth, 388

Zahn, J.-P. 1975, A\&A, 41, 329

Zahn, J.-P. 1977, A\&A, 57, 383 